\title{
Near patient testing for influenza in children in primary care: comparison with laboratory test
}

\author{
Anthony Harnden, Angela Brueggemann, Sasha Shepperd, Judy White, Andrew C Hayward, \\ Maria Zambon, Derrick Crook, David Mant
}

Department of Primary Health Care, Institute of Health Sciences, University of Oxford, Oxford OX3 7LF

Anthony Harnden university lecturer Sasha Shepperd university research lecturer

Judy White research nurse

David Mant professor and head of departmen

Academic

Department of

Microbiology and

Infectious Disease,

University of

Oxford, Oxford

OX3 9DU

Angela

Brueggemann

senior research fellow

Derrick Crook

consultant

microbiologist

University College

London Centre for

Infectious Disease

Epidemiology,

London NW3 2PF

Andrew C Hayward

senior lecturer

Virus Reference

Division, Central

Public Health

Laboratory,

Colindale, London

NW9 5HT

Maria Zambon

consultant virologist

Correspondence to:

A Harnden

anthony.harnden@

dphpc.ox.ac.uk

BMJ 2003;326:480
Influenza is an important cause of acute respiratory illness in young children. Common complications include febrile convulsions, otitis media, bronchiolitis, and croup. In epidemic years attack rates among preschool children often exceed $40 \%$. During these years children with influenza may account for up to $30 \%$ of the increase in antibiotic prescribing. ${ }^{1}$ Symptoms and signs of influenza in children are not specific and can mimic a range of other common respiratory viral pathogens. One quick way of reaching a precise diagnosis in primary care is to use a near patient test. Near patient testing for many conditions has expanded widely in primary care, though many tests have not been rigorously evaluated. ${ }^{2}$

Previous studies in children have compared near patient influenza tests with viral culture analysis using throat or nasal swabs. ${ }^{3}$ However, a nasopharyngeal aspirate is the best specimen for detecting influenza viruses, and polymerase chain reaction (PCR) is more sensitive than tissue culture when serology is the reference standard. ${ }^{4}$ We compared a near patient influenza test in children in primary care with laboratory based reverse transcription PCR (RT-PCR) testing of nasopharyngeal aspirates.

\section{Participants, methods, and results}

From January to March 2001 and October to March 2002 we asked general practitioners in Oxfordshire to identify children with cough and fever who they thought had more than a simple cold. Using a nasal swab we performed a near patient test for influenza (QuickVue; Quidel, San Diego, CA). A research nurse did the test, which took 12 minutes.

We collected a nasopharyngeal aspirate from the other nostril and transported the sample to the laboratory within four hours. The laboratory staff were blind to the result of the near patient test. After adding phosphate buffered saline to the aspirate we added the emulsified sample to viral lysis buffer before freezing it at $-80^{\circ} \mathrm{C}$. We used RT-PCR to convert the extracted nucleic acids from RNA to complementary DNA. We performed a multiplex, nested PCR assay, using primer sets specific to influenza $\mathrm{A}$ and $\mathrm{B}$, on all the samples. To validate our results we included quantified tissue culture specimens of influenza A and B as positive controls and water as negative control with every batch of samples tested.

A nasal swab and a nasopharyngeal aspirate were taken from 157 children. The children's median age was 3 years (range 6 months to 12 years), and 100 were boys. We detected influenza by RT-PCR in 61 children $(39 \%)$. The near patient test was positive in 27 of these 61 children, giving a sensitivity of $44 \% \quad(95 \%$ confidence interval $32 \%$ to $58 \%$ ) and a specificity of $97 \%(91 \%$ to $99 \%)$ (table). The likelihood ratio for a positive test result was 14.2 (4.5 to 44.7 ) and for a negative result 0.58 (0.46 to 0.72 ).
Comparison of near patient testing with reverse transcription polymerase chain reaction (RT-PCR) testing for influenza in children

\begin{tabular}{lccc} 
& \multicolumn{2}{c}{ RT-PCR test } & \\
\cline { 2 - 3 } & Positive & Negative & Total \\
\hline Near patient test: & & & \\
\hline Positive & 27 & 3 & 30 \\
\hline Negative & 34 & 93 & 127 \\
\hline Total & 61 & 96 & 157
\end{tabular}

\section{Comment}

The high specificity of this near patient test, combined with its ease of use, makes it suitable to "rule in" diagnosis of influenza in children in primary care, although its low sensitivity means it cannot "rule out" influenza. A sensitivity lower than has been described previously can be explained by our choice of RT-PCR as our reference standard, on a nasopharyngeal aspirate, rather than tissue culture testing on a nasal swab. ${ }^{3}$ Future evaluations of near patient tests should use molecular reference standards rather than traditional culture based techniques. A secure diagnosis of influenza in children in primary care may be important in guiding the general practitioner's optimal management, improving the surveillance of influenza, and satisfying parents, rather than telling them, "It's just a virus."

We thank the 40 Oxfordshire general practitioners who took part and Tim Lancaster for comments on an earlier draft of the paper.

Contributors: AH, ACH, DC, MZ, and DM designed the study. $\mathrm{AH}$ and $\mathrm{JW}$ took part in the fieldwork. $\mathrm{AB}, \mathrm{DC}$, and $\mathrm{MZ}$ were responsible for the laboratory work. AH and SS did the analysis. AH drafted the manuscript, and all authors commented on the text. AH is guarantor for the study.

Funding: The study was funded by the Medical Research Council as part of a programme grant in childhood infection in primary care (G0000340). The guarantor accepts full responsibility for the conduct of the study, had access to the data, and controlled the decision to publish.

Competing interests: None declared.

Ethical approval: Oxford clinical research ethics committee (C00.180).

1 Neuzil KM, Mellen BG, Wright PF, Mitchel EF Jr, Griffin MR. The effect of influenza on hospitalizations, outpatient visits, and courses of antibiotics in children. N Engl J Med 2000;342:225-31.

2 Delaney BC, Hyde CJ, McManus RJ, Wilson S, Fitzmaurice DA, Jowett S, et al. Systematic review of near patient test evaluations in primary care. BMJ 1999;319:824-7.

3 Rodriguez WJ, Schwartz RH, Thorne MM. Evaluation of diagnostic tests for influenza in a pediatric practice. Pediatr Infect Dis J 2002;21:193-6.

4 Carman WF, Wallace LA, Walker J, McIntyre S, Noone A, Christie P, et al. Rapid virological surveillance of community influenza infection in general practice. BMJ 2000;321:736-7.

5 Zambon M. Laboratory diagnosis of influenza. In Nicholson KG, Webster RG, Hay AJ, eds. Textbook of influenza. Oxford: Blackwell Science, 1998: 291-313.

(Accepted 17 January 2003) 